\title{
Determination of Critical Deformation Regions of a Lithium Polymer Battery by DIC Measurement and WOWA Filter
}

\author{
Szabolcs Kocsis Szürke ${ }^{1}$, Adrienn Dineva ${ }^{1}$, Szabolcs Szalai ${ }^{2}$, \\ István Lakatos ${ }^{3}$
}

${ }^{1}$ Research Center of Vehicle Industry, Széchenyi István University, Egyetem tér 1, H-9026 Györ, Hungary, kocsis.szabolcs@ga.sze.hu, dineva.adrienn@sze.hu

${ }^{2}$ Department of Vehicle Manufacturing, Széchenyi István University, Egyetem tér 1, H-9026 Györ, Hungary, szalaisz@sze.hu

${ }^{3}$ Department of Automotive and Railway Engineering, Széchenyi István University, Egyetem tér 1, H-9026 Györ, Hungary, lakatos@sze.hu

Abstract: This paper considers the determination method of deformation location of lithium polymer batteries. Measurements are performed using the Digital Image Correlation (DIC) technique and the obtained results are sorted into a database as a function of the charge level. A statistically based algorithm is used to eliminate measurement errors and outliers. This paper adopts the Weighted Ordered Weighted Averaging (WOWA) operator-based $2 D$ filtering method with the purpose of determining the critical regions of the cell. During the tests, several lithium polymer batteries of the same type but in different states are compared. Measurements on completely new and also on worn-out batteries are performed. The results support that the regions where greater deformation is expected during charging and discharging can be predicted. Results of investigations validate that the proposed approach is suitable for determining the critical deformation regions with high accuracy.

Keywords: battery swelling; battery testing; lithium polymer battery; GOM Atos; DIC measurement; WOWA

\section{Introduction}

Lithium batteries are currently widely used as a popular energy storage device in the automotive industry and among portable electronic devices as well. The main arguments in favor of them are high energy density and long stable operation. In the past years, lithium-based batteries have been getting widespread because of the increasing demand, financial investments and technological advantages. 
However, there are several developments and operational issues with their use, such as minimizing these faults, which is important for users. Numerous studies have also dealt with the state of charge (SOC) and state of health (SOH) estimation [1-4], temperature effect [5], or examination of cells at different pressures [43-45]. In addition to traditional tests, mechanical-based measurements have recently become popular, supplementing them to provide more accurate information on the internal state of lithium-ion batteries [6]. Studies have also been performed on cell impact [7-8], mechanical deformation [9] and pressure [10]. In addition to external effects, improper operation, overcharging [11], deep discharge, and a high number of cycles can lead to deformation and swelling of the cells. The main causes of this may be an expansion of the host materials, an expansion in the volume of the electrode, a change in pressure in the dead space of the cell, or gas formation [12-14]. In addition to continuous use, the number of cycles also increases, in which reversible volume change can also become an irreversible process. This involves a mechanical reaction of the battery cells, which can cause loss of capacity and failure. For a deeper analysis of structural properties, the reader is referred to [13] [15] [16] papers for additional details. Furthermore, continuous deformation can be observed even under normal, manufacturer-recommended use. In accordance with the State-of-the-Art, determining the critical location of this deformation is in the focus of this paper. Well-defined cell parameters and diameters are important factors in planning battery placement as well as $\mathrm{SOH}$ estimation. For this reason, measuring the deformation of lithium batteries has become a popular area of research in recent times. Several methods are used to measure deformation during use. Tactile tests measure the deformation of a cell at one or a few points, [17-21] it can even be tested together with the effect of pressure [22]. In most cases, a displacement sensor is used to measure them [23], placed in the middle of the battery, on this basis, a deformation map is even made [24]. The advantage of this method is that they can even examine the cells at the system level [25] and the deformation during storage continuously measurable [26]. The problem with this is that the battery varies asymmetrically and amorphously, making it difficult to determine where to measure. In addition, a number of good and useful publications have been produced with other sensors and methods of deformation measurement: thickness gauge [27], ultrasonic transducer pulser and receiver [28], strain sensor [29] or high resolution dilatometry [30]. The obtained data in force-SOC combination can even be used for charge-level estimation [31]. To better understand cells without destruction, CT scans are performed to look for structural defects [32] or to study the structural change of bad cells [33]. A detailed analysis of the methods used to measure deformation can be found in the following publications [34-35]. In this publication, we used the popular DIC technique for high-precision analysis of deformation. Thanks to its many benefits - such as easy experimental setups, simple implementation, high resistance to environmental influences, variability and widely adjustable time and space resolution - DIC technique has become widely accepted as a powerful and flexible tool for 
measuring the movement and deformation of different materials. With this solution, measurement accuracies of up to $+/-1-2 \mu \mathrm{m}$ can be achieved. The GOM system has been used successfully in a number of areas [36-37]. It is also used in the field of batteries, mostly for electrode composition testing [38] and structural characteristics measurement [39] but has also been used for deformation measurement [40-41] possibly in combination with other methods [42]. This paper is structured according to the following: The measurement process section describes the measurement procedure, tools, and data storage. In the third chapter, the adoption of the WOWA operator is presented. The evaluation and results section presents the measurement results using a filtering procedure.

\section{Measurement Process}

Intercalation between lithium batteries occurs during charging and discharging, not in no-load condition or a discharged state, thus, there is no or a very low degree of deformation during the interruption. This assumption was confirmed in our previous publication by results from displacement sensor measurements [46]. Consequently, tests can be interrupted and optical measurements can be made. In order to achieve greater accuracy and better mapping of critical locations, the tests were performed using DIC technique. The meaning of a DIC technique is Digital Image Correlation. According to the literature with this technique can be measure displacement, deformation, 3D coordinates and can be made 3D scanning. In this article, a 3D coordinates measurement was used with a 3D scanning DIC system. The DIC system was the GOM Atos TripleScan II hardware with GOM Atos Professional software. The ATOS Triple Scan non-contact structured blue light $3 \mathrm{D}$ scanner is a type of coordinate measuring machine that measures millions of points per single scan/measurement. It uses advanced measuring and projection techniques to produce high quality data and precision accuracy for full-object dimensional analysis. ATOS sensors are self-monitoring systems. The sensors identify changing ambient conditions during operation. The software of the sensors is continuously monitoring the calibration status, the transformation accuracy as well as environmental changes and part movements in order to ensure the quality of the measuring data. In this research the battery was measured several times and after that the individual measurements were assembled in the software. This allowed a detailed analysis of the deformation. Several conditions must be fulfilled for accurate measurement, therefore, the DIC technique requires the following:

- Reduce the reflection: The quality of preparation is important for DIC measurements. Thus, the object to be measured should not be reflective, otherwise, the test element should be thinly coated with special antireflex paint [47]. In this article, a MR2000 anti-reflex spray was used. (In our case, the surface had to be treated.) 
- Use reference points: Another important thing is that the camera detects at least three reference points during the measurement. The GOM Atos system uses special coded reference points in several sizes. For the measurements was used $1 \mathrm{~mm}$ and $0.8 \mathrm{~mm}$ coded reference points.

- Taking pictures: In these tests, we used the two-camera GOM ATOS measuring unit, where one camera digitizes based on the reference points and the other functions as a control.

- Image analysis: After recording, the deformation images are compared to a reference or initial image using a special cross-correlation algorithm that will extract the displacement fields.

During the tests, the following devices were used to determine the electrical parameters: the power supply - Hameg HMP 4030, the dummy load - EL3000, the data acquisition card - NI 9201 and lithium polymer battery - Turnigy nano-tech LiPo $5 \mathrm{Ah}$. LabView software is responsible for test control. The tests were performed on 3 different 5 Ah lithium polymer batteries. The cells were of the same in type, size and capacity, the only difference being the production time. A new and old battery was used during the measurements. In the following distribution:

- Battery number 1 (approximately 80\% capacity): charging $4.2 \mathrm{~V}$ and discharging up to $3 \mathrm{~V}$.

- Battery number 1 (6 months later):

○ Charging $4.2 \mathrm{~V}$ and discharging up to $3 \mathrm{~V}$.

- Charging $4.2 \mathrm{~V}$ and discharging up to $0 \mathrm{~V}$.

- Battery number 2 (approximately 30\% capacity): charging $4.2 \mathrm{~V}$ and discharging up to $0 \mathrm{~V}$.

- Battery number 3 (approximately $100 \%$ capacity): charging $4.2 \mathrm{~V}$ and discharging up to $3 \mathrm{~V}$.

A total of 5 charge and 5 discharge tests were performed. The general course of the tests was as follows: fully charge the battery $100 \%$-SOC; digitization of a fully charged state; start discharging and interrupt, digitize every $360 \mathrm{~s}$; in general, surface measurements are made: $100 \%, 90 \% \ldots 10 \%$ and $0 \%$ of the charge level; conditioning for one hour; re-digitization of a fully discharged state; start charging and interrupt, digitize every $360 \mathrm{~s}$. In general, surface measurements are made at $0 \%, 10 \% \ldots 90 \%$ and $100 \%$ of the charge level. After battery replacement, these steps were performed on each cell. 

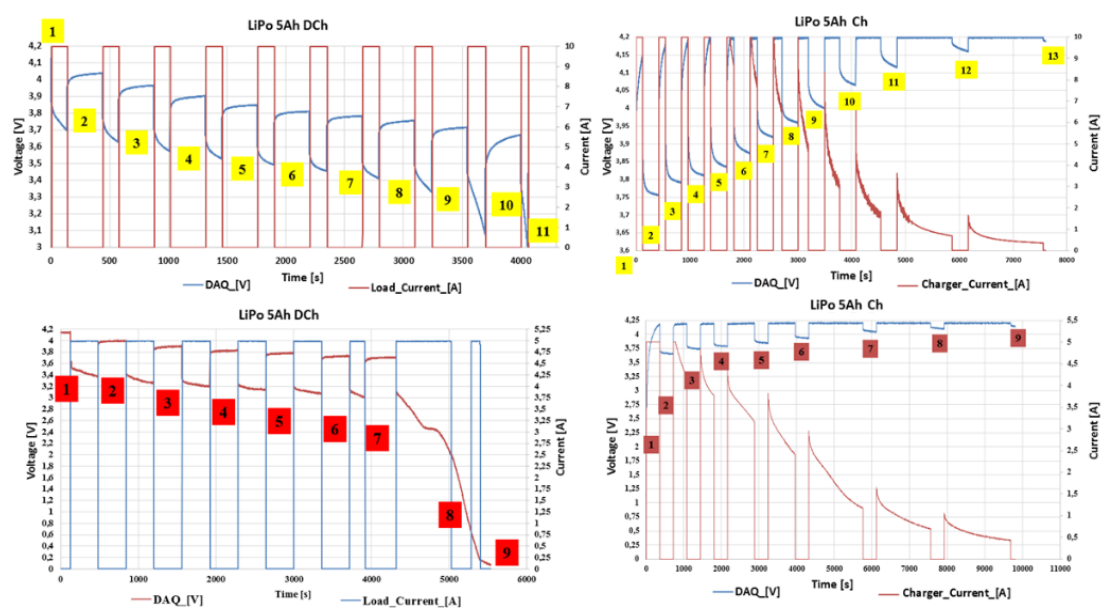

Figure 1

Charging and discharging characteristics of LiPo batteries

Figure 1 shows the charging and discharging current and voltage profile of a normal $(3 \mathrm{~V})$ and a fully discharged $(0 \mathrm{~V})$ cell. The upper part of the Fig. 1 shows the voltage / current diagrams obtained during the first test, from the left during discharge and from the right during charging. The bottom of the Fig. 1 shows the results of cell number two, in this test we discharged the battery to $0 \mathrm{~V}$. In this case, too, the voltage / current profiles measured during discharge are shown on the left and the charging on the right. The numbering shown in the Fig. 1 can be observed at interruptions, digitization has taken place at these points. Figure 1 shows that the number of interrupts varied as a function of the length of the tests. For example, the upper right Fig. 1 had 9 interrupts, representing 11 images (including the start and end states), and the lower left figure had 7 interrupts, representing 9 images. The reason for this is that the battery discharges faster. The number of digitization points created during charging also varies, which are due to the faster charging of the cell or optimization of the measurement time. In some cases, at the end of the charge, we digitized every 10 to 20 minutes because in this case the charged energy is less, so the degree of deformation is smaller based on the observations. Furthermore, in each case we store the data depending on the charge level, therefore, it will not cause an issue with a different number of digitization points. Figure 2 shows the results of four measurements, in each case also showing the A (on the right side of the picture pair) and B (on the left side of the picture pair) side of the battery. The cells change asymmetrically and amorphously. In general, they swell during charging and contract during discharge. Figure 2 shows the largest deviation from the initial state, which is $100 \%$ SOC during charging and 0\% SOC during discharge. The lower left corner shows the results during charging, and the lower right corner shows the fully discharged state up to $0 \mathrm{~V}$. Based on these few deep discharges, it was observed that in the range below $3 \mathrm{~V}$, contraction is replaced by swelling. 


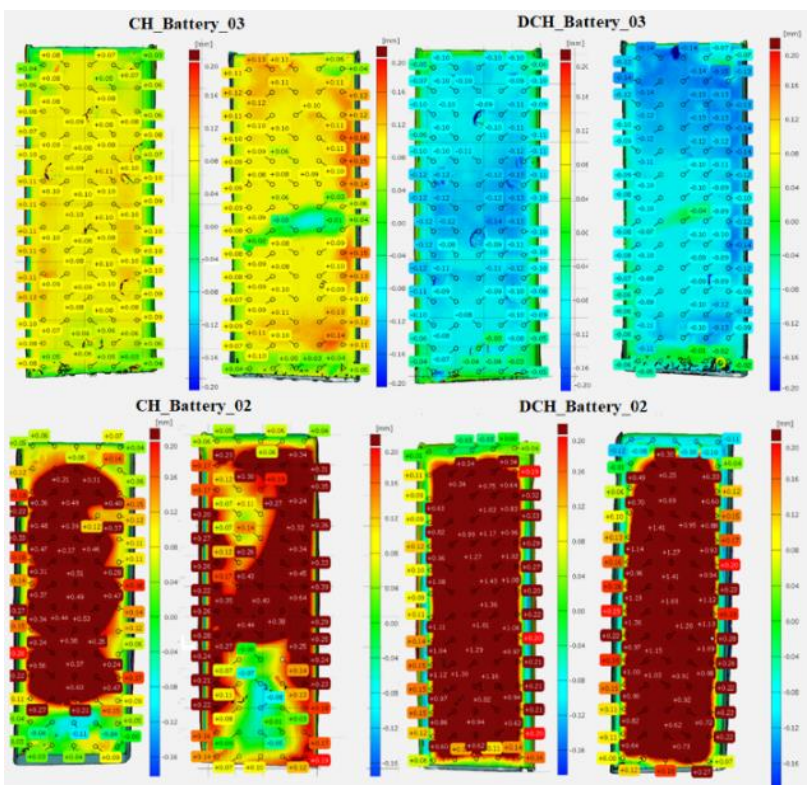

Figure 2

Deformation images recorded using the DIC technique

All electrical and surface digitization points are stored together and arranged in data matrices depending on the charge level.
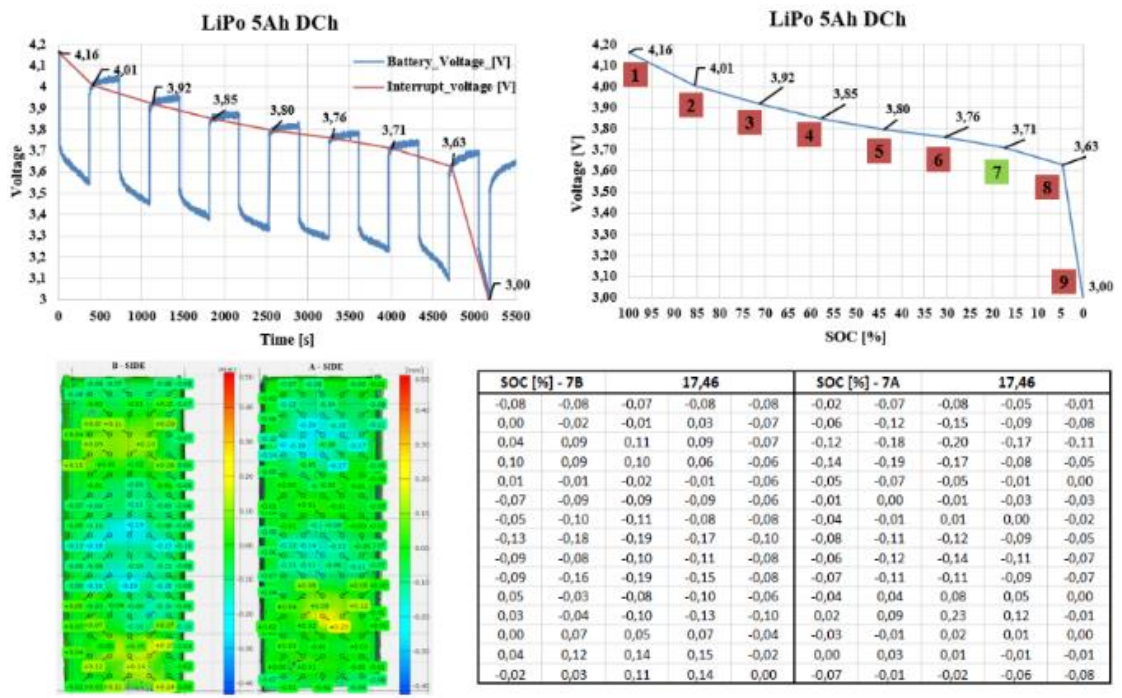

\begin{tabular}{|c|c|c|c|c|c|c|c|c|c|}
\hline \multicolumn{2}{|c|}{$\operatorname{SOC}[\%]-7 B$} & \multicolumn{3}{|c|}{17,46} & \multicolumn{2}{|c|}{$50 C[3]-7 A$} & \multicolumn{3}{|c|}{17,46} \\
\hline-0.08 & 0.08 & -0.07 & -0.08 & -0.08 & $-0,02$ & -0.07 & -0.08 & 0.05 & 0.01 \\
\hline 0,00 & $-0,02$ & $-0,01$ & 0,03 & $-0,07$ & $-0,06$ & $-0,12$ & $-0,15$ & $-0,09$ & $-0,08$ \\
\hline 0.04 & 0.09 & 0.11 & 0.09 & -0.07 & $-0,12$ & -0.18 & -0.20 & .0 .17 & -0.11 \\
\hline 0,10 & 0.09 & 0,10 & 0.06 & -0.06 & $-0,14$ & -0.19 & -0.17 & $-0,08$ & $-0,05$ \\
\hline 0.01 & $-0,01$ & $-0,02$ & & $-0,06$ & $-0,05$ & $-0,07$ & $-0,05$ & $-0,01$ & 0,00 \\
\hline$-0,07$ & $-0,09$ & -0.09 & $-0,09$ & $-0,06$ & $-0,01$ & 0,00 & -0.01 & $-0,03$ & $-0,03$ \\
\hline$-0,05$ & $-0,10$ & -0.11 & $-0,08$ & $-0,08$ & $-0,04$ & -0.01 & 0,01 & 0,00 & $-0,02$ \\
\hline$-0,13$ & $-0,18$ & -0.19 & $-0,17$ & $-0,10$ & $-0,08$ & $-0,11$ & $-0,12$ & $-0,09$ & -0.05 \\
\hline$-0,09$ & $-0,08$ & $-0,10$ & $-0,11$ & $-0,08$ & $-0,06$ & $-0,12$ & $-0,14$ & $-0,11$ & $-0,07$ \\
\hline$-0,09$ & 0,16 & $-0,19$ & 0,15 & $-0,08$ & $-0,07$ & $-0,11$ & $-0,11$ & 0,09 & 0,07 \\
\hline 0,05 & $-0,03$ & $-0,08$ & $-0,10$ & $-0,06$ & $-0,04$ & 0,04 & 0,08 & 0,05 & 0,00 \\
\hline 0,03 & $.0,04$ & 0.10 & 0,13 & 0,10 & 0,02 & 0,09 & 0.23 & 0,12 & 0,01 \\
\hline 0,00 & 0,07 & 0,05 & 0,07 & $-0,04$ & $-0,03$ & $-0,01$ & 0,02 & 0,01 & 0,00 \\
\hline 0,04 & 0,12 & 0,14 & 0,15 & $-0,02$ & 0,00 & 0,03 & 0,01 & $-0,01$ & 0,01 \\
\hline$-0,02$ & 0,03 & 0,11 & 0,14 & 0,00 & -0.07 & -0.01 & 0,02 & $-0,06$ & 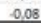 \\
\hline
\end{tabular}

Figure 3

Storing surface digitization data in a database 
Figure 3 shows the data aggregation. The results during the discharge of cell 2 can be seen in the upper left image of the Fig. 3, in addition to the voltage diagram, the points of the interruptions are also shown. In the upper right image the same points are shown, but already as a function of the charge level. The Coulomb Counting method was used to determine the charge level. The digitization point 7 is marked in green on the diagram, which is shown in detail in the lower part of Fig. 3. The lower left image displays the digitization data and the lower right image shows the data matrix as a function of the charge level.

\section{Adoption of WOWA Operator for Critical Region Detection}

From the millions of measurement points created during digitization, we selected 75-80 measurement sites evenly spaced and stored them in the data matrix. The recording of equally spaced points is automatically networked and recorded by the GOM measuring system software. It is important to note that the same measurement uses the same mesh for all charge levels. There is a relatively short time (5 minutes) available for digitization during discharge and charge interruptions, so in some cases, errors may occur. Therefore, smaller holes are created where there is no accurate deformation information and incorrect data can be entered here. Therefore, the results should be evaluated by a statistical method in order to effectively filter the database from errors and outliers. Several methods are used in the literature for similar problems [48-51]. Torres et al. present an efficient method for post-filtering images using the WOWA-operator [52], whose applicability in critical region determination is considered in this paper.

\subsection{Mathematical Background of Aggregation Operators}

The need of information fusion has become increasingly important in various disciplines of modern engineering and artificial intelligence [53]. The aggregation functions are mathematical functions that are used to incorporate various information. The arithmetic mean and the weighted mean are the most wellknown aggregation operators. The main characteristic of the weighted mean is that it permits us to weight the different data according to their relevance that is not possible with the arithmetic mean. The arithmetic mean is given in Eq. (1).

$$
W^{A M}\left(a_{1}, \ldots, a_{n}\right)=\sum_{k=1}^{n} \frac{a_{k}}{n}
$$

The weighted mean can be formulated as follows: 


$$
W^{W M}\left(a_{1}, \ldots, a_{n}\right)=\sum_{i=1}^{n} w_{i} a_{i}
$$

where $\mathrm{w}_{\mathrm{i}}$ stand for the weights of the $\mathrm{k}^{\text {th }}$ data source. The weights are positive and $\sum w_{i}=1$. The classical aggregation operators such the weighted average, are key tools of decision theory [54]. However, nowadays they are considered particular families of more general aggregation operators. Originally, the possible aggregation of fuzzy sets required operations that provide a single fuzzy number as a result of combining several fuzzy numbers [55]. Many fuzzy aggregation operators have been presented, such as the product, t-norms, different types of means, etc. The Ordered Weighted Averaging (OWA) operator has been introduced in [56] by Yager, that is a class of generalized mean operator. The OWA operator has the form [56]:

$$
W^{\text {OWA }}\left(a_{1}, \ldots, a_{n}\right)=\sum_{i=1}^{n} w_{i} a_{\sigma(i)}
$$

in which $\sigma(\mathrm{i})$ corresponds to the permutation of $\mathrm{a}_{\mathrm{i}}$, i.e. the elements of the data vector are sorted decreasing order, from the largest value to the lowest one. This sorting allows to give the desired importance to the largest, lowest or medium value data. Several papers have been published about the properties and modifications of aggregation operators [57]. It is worth noting that OWA aggregation functions and weighted arithmetic means are special cases of Choquet integral. A large family of aggregation functions based on Choquet integrals [58]. Their detailed properties and definition are given with respect to a fuzzy measure [57]. When the fuzzy measure is additive, Choquet integrals become weighted arithmetic means, and when the fuzzy measure is symmetric, they become OWA functions. Different OWA operators are distinguished in the literature according to their weighting function. In [59] Torra has introduced the Weighted OWA (WOWA) aggregation function, that can be considered as the generalization of weighted means. The weighted OWA function has two sets of weights, one of them has the same function as the weighting vector in weighted means, wherease the other is equivalent to the weighting vector in OWA.

\subsection{Outline of the Proposed Method}

Due to its excellent properties the WOWA operator has wide application possibilities. For instance, image processing techniques can benefit from such an approach. The efficiency of this method is that two vectors can be used to well set the importance of data and filter out erroneous data. The other thing that makes a statistically based analysis useful is that the differences between points close to each other can be well filtered. The goal with these measurements is to determine 
a critical region, so outliers can make this very difficult. The first step in processing the data is to read the database and rearrange it using the algorithm:

\begin{tabular}{|c|c|c|c|c|}
\hline \multicolumn{2}{|c|}{ SOC [\%] - 9B } & \multicolumn{3}{c|}{$\mathbf{0 , 0 0}$} \\
\hline$-0,04$ & $-0,10$ & $-0,12$ & $-0,07$ & $-0,03$ \\
$-0,09$ & $-0,18$ & $-0,19$ & $-0,13$ & $-0,11$ \\
\hline$-0,16$ & $-0,23$ & $-0,25$ & $-0,22$ & $-0,15$ \\
\hline$-0,18$ & $-0,24$ & $-0,24$ & $-0,14$ & $-0,07$ \\
\hline$-0,08$ & $-0,11$ & $-0,10$ & $-0,04$ & $-0,02$ \\
\hline$-0,05$ & $-0,02$ & $-0,04$ & $-0,06$ & $-0,05$ \\
\hline$-0,06$ & $-0,03$ & $-0,02$ & $-0,03$ & $-0,03$ \\
\hline$-0,11$ & $-0,14$ & $-0,16$ & $-0,12$ & $-0,07$ \\
\hline$-0,09$ & $-0,17$ & $-0,20$ & $-0,15$ & $-0,09$ \\
\hline$-0,10$ & $-0,16$ & $-0,16$ & $-0,14$ & $-0,10$ \\
\hline$-0,07$ & 0,00 & 0,04 & 0,02 & $-0,02$ \\
\hline 0,00 & 0,05 & 0,20 & 0,09 & $-0,02$ \\
\hline$-0,06$ & $-0,06$ & $-0,03$ & $-0,03$ & $-0,02$ \\
\hline$-0,01$ & 0,00 & $-0,02$ & $-0,03$ & $-0,02$ \\
\hline$-0,10$ & $-0,03$ & $-0,05$ & $-0,08$ & $-0,10$ \\
\hline
\end{tabular}

Figure 4

Rearrange the database

In the figure, the point to be determined is marked in yellow, and the data of the 3 $\mathrm{X} 3$ matrix marked in blue were used for the determination. Based on this, a total of 9 values were used to determine a selected point (marked in yellow). The following relationship describes the coordinate points of the data extracted from the matrix:

$$
\begin{array}{ccc}
\mathrm{I}(\mathrm{x}-1 ; \mathrm{y}-1) & \mathrm{I}(\mathrm{x}-1 ; \mathrm{y}) & \mathrm{I}(\mathrm{x}-1 ; \mathrm{y}+1) \\
\mathrm{I}(\mathrm{x} ; \mathrm{y}-1) & \mathrm{I}(\mathrm{x} ; \mathrm{y}) & \mathrm{I}(\mathrm{x} ; \mathrm{y}+1) \\
\mathrm{I}(\mathrm{x}+1 ; \mathrm{y}-1) & \mathrm{I}(\mathrm{x}+1 ; \mathrm{y}) & \mathrm{I}(\mathrm{x}+1 ; \mathrm{y}+1)
\end{array}
$$

The data is transferred from the matrix to the row vector according to the following formula:

$$
\begin{aligned}
& \begin{array}{lll}
I_{3} & I_{6} & I_{9}
\end{array}
\end{aligned}
$$

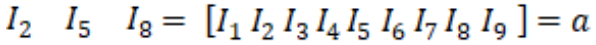

$$
\begin{aligned}
& \begin{array}{lll}
I_{1} & I_{4} & I_{7}
\end{array}
\end{aligned}
$$

When determining weight vectors, all values were considered to be of equal significance.

$$
p=[1 / 9 ; 1 / 9 ; 1 / 9 ; 1 / 9 ; 1 / 9 ; 1 / 9 ; 1 / 9 ; 1 / 9 ; 1 / 9]
$$

An empirically determined weight vector can be further investigated if even finer critical region detection performance is required by emphasizing distinct parts of the battery. When specifying the second vector, our purpose is to eliminate, filter out outliers and highlight critical regions.

$$
W_{n}=[0 ; 0,075 ; 0,125 ; 0,175 ; 0,250 ; 0,175 ; 0,125 ; 0,075 ; 0]
$$


As a first step, we determined the coordinate points based on the following formula:

$$
\begin{gathered}
\left(i / n ; \sum_{j \leq i} \omega_{j}\right) \\
i=1\left(1 / 9 ; \omega_{1}\right)(1 / 9 ; 0) \\
i=2 \quad\left(2 / 9 ; \omega_{1}+\omega_{2}\right)(2 / 9 ; 0,075) \\
i=3 \quad\left(8 / 9 ; \omega_{1}+\omega_{2}+\omega_{3}\right)(1 / 3 ; 0,2) \\
i=4 \quad\left(4 / 9 ; \omega_{1}+\omega_{2}+\omega_{3}+\omega_{4}\right) \quad(4 / 9 ; 0,375) \\
i=6 \quad\left(6 / 9 ; \omega_{1}+\omega_{2}+\omega_{2}+\omega_{3}+\omega_{4}+\omega_{5}\right) \quad(5 / 9 ; 0,625) \\
i=8 \quad\left({ }^{7} / 9 ; \omega_{1}+\omega_{2}+\omega_{3}+\omega_{4}+\omega_{5}+\omega_{6}+\omega_{7}\right) \quad(7 / 9 ; 0,925) \\
\left.i=9 \omega_{6} ; \omega_{1}+\omega_{2}+\omega_{3}+\omega_{4}+\omega_{5}+\omega_{6}+\omega_{7}+\omega_{8}\right) \quad(8 / 9 ; 1)
\end{gathered}
$$

After curve fitting, the following function was obtained:

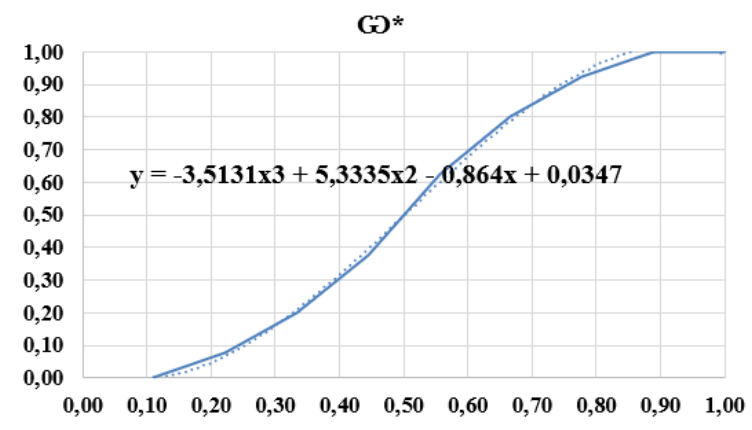

Figure 5

Determined $\omega^{*}$ after curve fitting

The equation after fitting the curve is as follows:

$$
\omega^{*}(x)=-3,513 x^{3}+5,3335 x^{2}-0,864 x+0,0347
$$


Using $\omega^{*}(\mathrm{x})$ we determined the weights:

$$
\begin{gathered}
i=1 \omega_{1}=\omega^{*}\left(p_{1}\right) \cong 0 \\
i=2 \quad \omega_{2}=\omega^{*}\left(\sum_{i=1}^{2} p_{i}\right)-\omega^{*}\left(p_{1}\right) \cong 0,068 \\
i=3 \quad \omega_{3}=\omega^{*}\left(\sum_{i=1}^{3} p_{i}\right)-\omega^{*}\left(\sum_{i=1}^{2} p_{i}\right) \cong 0,142 \\
i=4 \quad \omega_{4}=\omega^{*}\left(\sum_{i=1}^{4} p_{i}\right)-\omega^{*}\left(\sum_{i=1}^{3} p_{i}\right) \cong 0,187 \\
i=5 \quad \omega_{5}=\omega^{*}\left(\sum_{i=1}^{5} p_{i}\right)-\omega^{*}\left(\sum_{i=1}^{4} p_{i}\right) \cong 0,206 \\
i=6 \quad \omega_{6}=\omega^{*}\left(\sum_{i=1}^{6} p_{i}\right)-\omega^{*}\left(\sum_{i=1}^{5} p_{i}\right) \cong 0,187 \\
i=7 \quad \omega_{7}=\omega^{*}\left(\sum_{i=1}^{7} p_{i}\right)-\omega^{*}\left(\sum_{i=1}^{6} p_{i}\right) \cong 0,142 \\
i=8 \quad \omega_{8}=\omega^{*}\left(\sum_{i=1}^{8} p_{i}\right)-\omega^{*}\left(\sum_{i=1}^{7} p_{i}\right) \cong 0,068 \\
i=9 \quad \omega_{9}=\omega^{*}\left(\sum_{i=1}^{9} p_{i}\right)-\omega^{*}\left(\sum_{i=1}^{8} p_{i}\right) \cong 0
\end{gathered}
$$

The obtained weight vector is as follows:

$$
\omega=[0 ; 0,068 ; 0,142 ; 0,187 ; 0,206 ; 0,187 ; 0,142 ; 0,068 ; 0]
$$

The first step in evaluating the values was to load the data (heatmap of deformation $[\mathrm{mm}])$ :

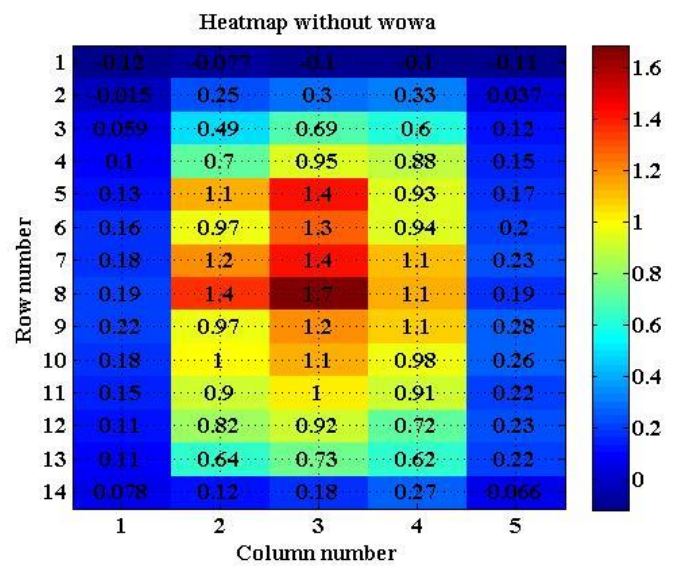

Figure 6

The last point of discharge is without the WOWA- based method 
The second step is to determine the corresponding $3 \mathrm{X} 3$ matrix for each data point based on Eq. (4). The determined values were then sorted in ascending order and applied to the following summary:

$$
f_{\text {WoWA }}=\sum_{i=1}^{9} \omega_{i} * a_{i}
$$

The final deformation point matrix was as follows:

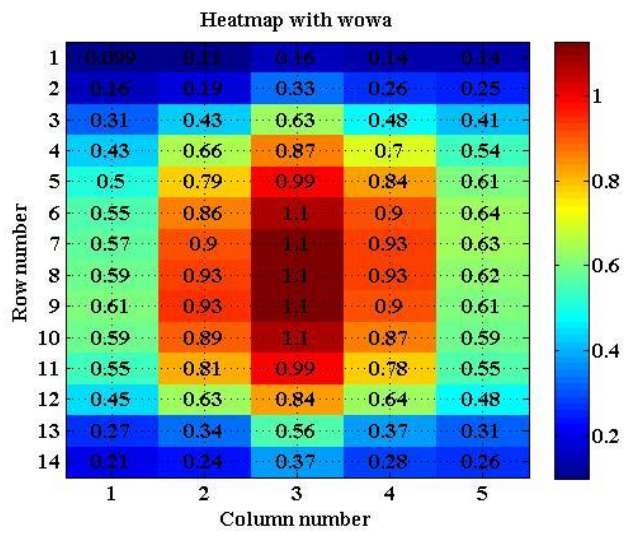

Figure 7

The last point of discharge after using the WOWA- based method

The values shown in Fig. 7 the dimension is [mm], the battery is divided into 5 columns and 14 rows. During the test, the battery was discharged to $0 \mathrm{~V}$. Based on the results, it can be concluded that this type of deep discharge causes significant swelling. Based on the heat map, it can be observed that the middle part of the cell changed the most. It can be observed that the filtered data eliminate outliers, possible errors and form a more uniform heat map. We also visualized the results for better presentation. Figure 8 shows a comparison of the measured and filtered values after visualization.

Visualization allows to better observe how the algorithm equalizes the surface of the battery. By eliminating the local minimum and maximum, the critical zone can be more easily selected. During this test, the greatest deformation occurred in the middle of the cell. More specifically, points 6-7-8-9-10 in column 3 are the most critical. 

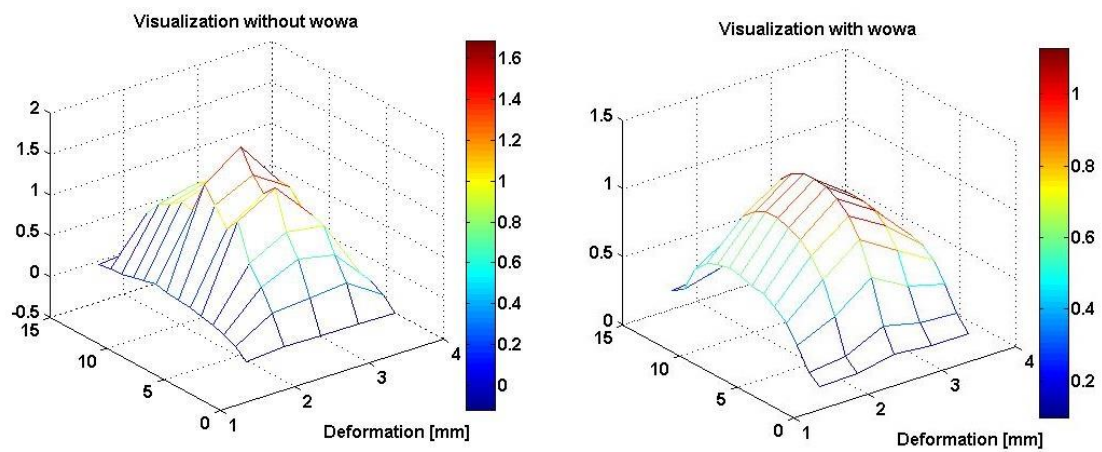

Figure 8

Visualization of the results obtained with the WOWA- based method

\section{Evaluation and Results}

Using the WOWA-based method, we filtered all states from outliers and potentially erroneous data and created a unified deformation map. To determine the critical regions, all digitization states and measured points of each measurement had to be examined. During the analysis, we observed that the examined point was smaller or larger than the average deformation point, based on this, it was classified as 1 or 0 . The average deformation point, in this case, is the average of the data on the unchanged side of the cell measured at the same charge level. In all cases, the value 1 represents the critical deformation greater than average. Values higher than average during charging and lower values during discharge were considered 1 because contraction is expected during discharge. Figure 9 shows the results obtained when charging side B of battery number 2 in the case of $100 \%$ SOC.

The upper part of the Fig. 9 shows the analyzed data, the left image shows the original data, and the right image shows the filtered results. The bottom of the Fig. 9 shows the results of the under/over test, the original data is shown in the left image (mean value $0.109 \mathrm{~mm}$ ) and the results filtered by the WOWA algorithm are shown in the right image (mean value $0.108 \mathrm{~mm}$ ). The next step in the analysis was to fit the data to each other, hence to summarize the critical points of the measurements for the same measurement and side (separately for A and B). 

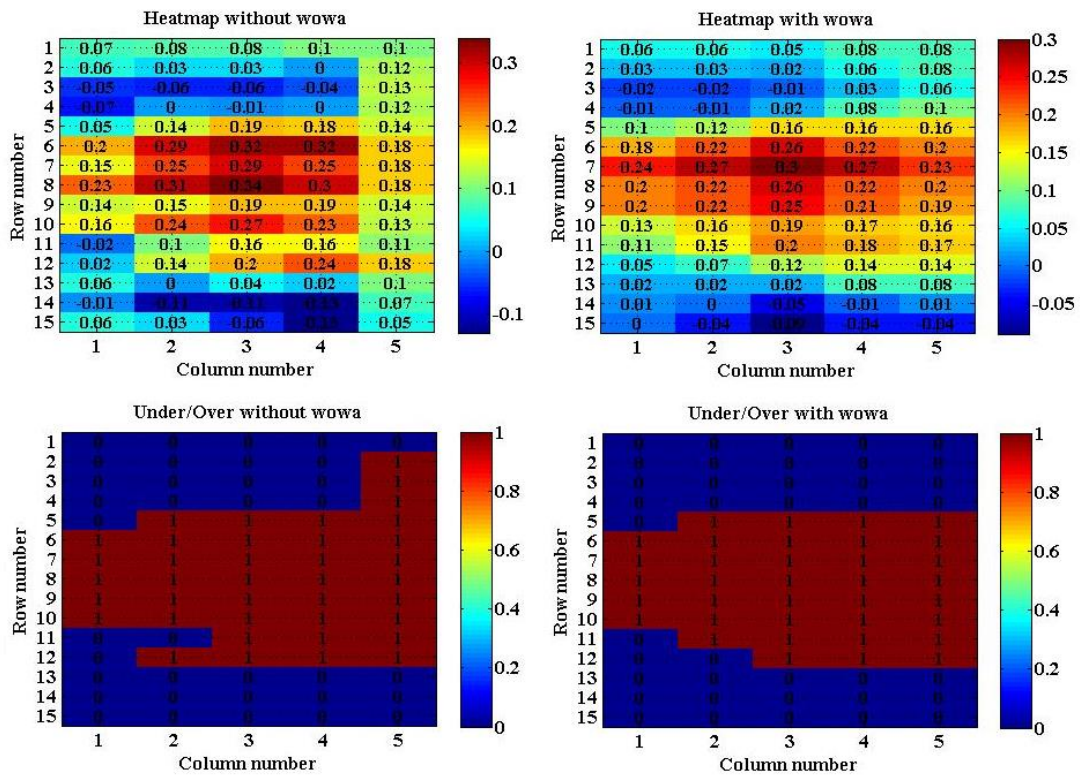

Figure 9

Deformation average test

In Fig. 10 shows the results obtained after data alignment:
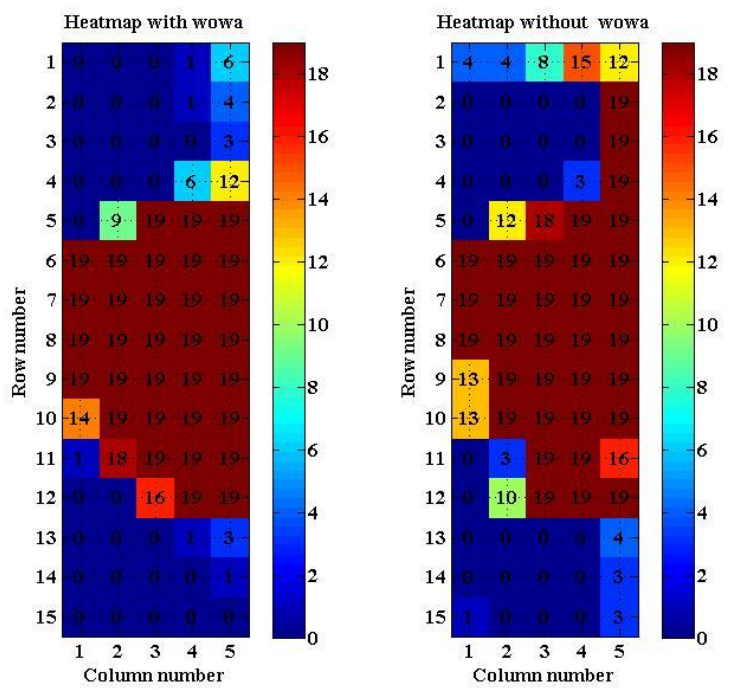

Figure 10

Deformation average test during charge (battery side B) 
It can be observed there were a total of 19 deformation states during this test and that the upper and middle parts of the battery changed the most during these measurements. The original data is shown on the left and the results after filtering are shown on the right. It can be seen that the critical points and regions are better concentrated with the filtering. The differences between sides A and B of the same test can be observed in the following in Fig. 11:
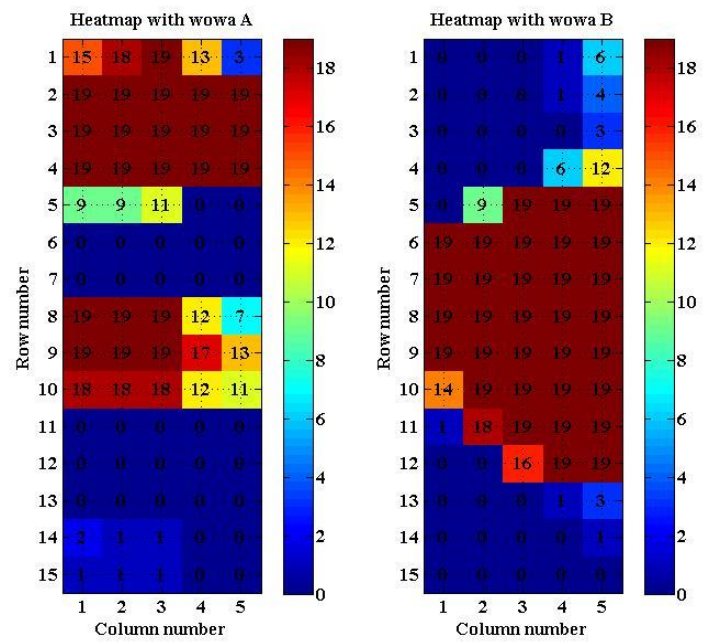

Figure 11

Deformation average test during charge (battery side A and B)

Figure 11 shows the filtered data by using WOWA-based method. We have already established that the cell sides change asymmetrically, and these results support this assumption. In this measurement, the lower part of the battery is the least critical region. In real cases, it is difficult to distinguish exactly which side A and B of the cell are, therefore, the results should be combined. Figure 12 shows the results of merging the two sides during charging and discharging, expressed as a percentage.

Figure 12 shows a charge/discharge cycle for the same battery. The results are similar, but there are also differences. The similarity is that in both cases, 8-9 rows in the middle of the battery, the most critical region, and the bottom of the battery were the least important. The deviation is observed in the upper part of the cell, during discharge, the upper right image became a highly critical area. To better assess the critical areas, all charging and discharging results were projected onto each other. Measurements from all 10 tests (5 charges, 5 discharges) from the three different batteries (sides A and B) were summarized. 



Figure 12

Deformation average test during charge and discharge (combined A and B)

The state after aggregating, averaging and sorting the data is as follows:


Figure 13

Critical regions of charging and discharging 
The combined results of all charge tests for all three cells are shown on the left side of the figure, and the results after discharge are shown on the right side. According to the result after charging, moving towards the middle of the cell, we get the critical areas, the lower and upper parts are less dominant. During discharge, the lower part is also less important, however, in this case, the critical region has shifted to the right and upwards. A summary of these results is required to determine a general case. In terms of cell criticality, the following 3 categories were defined: 0-non-critical (0-33\%), 1-moderately critical (33-66\%), 2-important region $(66-100 \%)$. The final step of the study is to combine the results and form a general classification picture. Figure 14 shows the last statement:
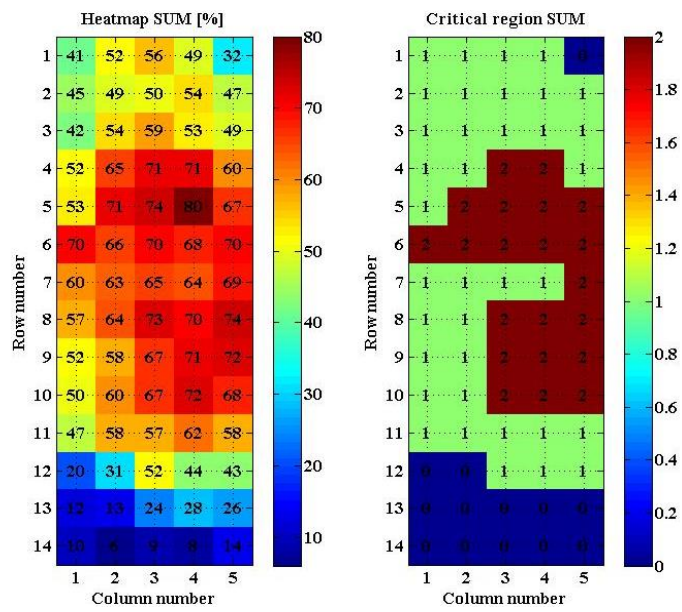

Figure 14

Categorized critical regions of the 5 Ah lithium polymer battery

Based on the images, it can be concluded that the critical regions occur the most common in the middle part of the cell since measurements should concentrate these regions. In the case of measuring in only one or a few points, it is more likely that critical region towards the right edge are found. Furthermore, it can be observed that the center of the cell is rarely the most ideal measuring point. Based on the analysis and calculations, it is advisable to place the displacement sensor above the middle, to the right, 5-6 rows 3-4 columns. The smallest deformation is probably to be measured at the bottom of the cell. In the case of conducting charge (see Fig. 13), test only, the middle part of the battery is the more ideal choice. In case of discharge (see Fig. 13), it is suggested to perform the measurement a little higher than the middle part.

\section{Conclusions}

Digitization of the battery surface is a new and state-of-the-art method for obtaining a very accurate data set on the deformation of cells during charging and discharging. Altogether of three lithium polymer battery measurements of the 
same type but different states are evaluated. The electrical and surface digitization points are aggregated and arranged in data matrices depending on the charge level. We propose the adoption of WOWA-based filtering method for the detection of critical regions. We effectively filtered the deformation database from errors and outliers, making it easier to identify critical points. Implementing the application of WOWA in a 2D convolution filter to a digital image correlation procedure presents new approach to a current engineering problem. Based on the results, it can be concluded that the method is suitable for filtering deformation data, and critical regions can be efficiently determined on the lithium polymer battery.

\section{Acknowledgment}

The research was supported by the Ministry of Innovation and Technology NRDI Office within the framework of the Autonomous Systems National Laboratory Program.

\section{References}

[1] D. N. T. How, M. A. Hannan, M. S. Hossain Lipu, and P. J. Ker, "State of Charge Estimation for Lithium-Ion Batteries Using Model-Based and DataDriven Methods: A Review," IEEE Access, Vol. 7, pp. 136116-136136, 2019

[2] A. Fotouhi, D. J. Auger, K. Propp, S. Longo, and M. Wild, "A review on electric vehicle battery modelling: From Lithium-ion toward LithiumSulphur,” Renew. Sustain. Energy Rev., Vol. 56, pp. 1008-1021, 2016

[3] Y. Wang et al., "A comprehensive review of battery modeling and state estimation approaches for advanced battery management systems," Renew. Sustain. Energy Rev., Vol. 131, No. July, p. 110015, 2020

[4] F. A. Shah, S. Shahzad Sheikh, U. I. Mir, and S. Owais Athar, "Battery health monitoring for commercialized electric vehicle batteries: Lithiumion," $5^{\text {th }}$ Int. Conf. Power Gener. Syst. Renew. Energy Technol. PGSRET 2019, no. August, pp. 1-6, 2019

[5] J. Yi, U. S. Kim, C. B. Shin, T. Han, and S. Park, "Three-Dimensional Thermal Modeling of a Lithium-Ion Battery Considering the Combined Effects of the Electrical and Thermal Contact Resistances between Current Collecting Tab and Lead Wire,” J. Electrochem. Soc., Vol. 160, No. 3, pp. A437-A443, 2013

[6] Mallarapu, A., Kim, J., Carney, K., DuBois, P., Santhanagopalan, S., Modeling Extreme Deformations in Lithium Ion Batteries, eTransportation

[7] Z. Pan, W. Li, and Y. Xia, "Experiments and 3D detailed modeling for a pouch battery cell under impact loading," J. Energy Storage, Vol. 27, No. August 2019, 2020

[8] J. Zhu, T. Wierzbicki, and W. Li, "A review of safety-focused mechanical modeling of commercial lithium-ion batteries," J. Power Sources, Vol. 378, no. November 2017, pp. 153-168, 2018 
[9] L. Greve and C. Fehrenbach, "Mechanical testing and macro-mechanical finite element simulation of the deformation, fracture, and short circuit initiation of cylindrical Lithium ion battery cells," J. Power Sources, Vol. 214, pp. 377-385, 2012

[10] V. Müller, R. G. Scurtu, M. Memm, M. A. Danzer, and M. WohlfahrtMehrens, "Study of the influence of mechanical pressure on the performance and aging of Lithium-ion battery cells," J. Power Sources, Vol. 440, no. May, p. 227148, 2019

[11] D. Ren, X. Feng, L. Lu, X. He, and M. Ouyang, "Overcharge behaviors and failure mechanism of lithium-ion batteries under different test conditions," Appl. Energy, Vol. 250, no. April, pp. 323-332, 2019

[12] C. R. Fell, L. Sun, P. B. Hallac, B. Metz, and B. Sisk, "Investigation of the Gas Generation in Lithium Titanate Anode Based Lithium Ion Batteries," J. Electrochem. Soc., Vol. 162, No. 9, pp. A1916-A1920, 2015

[13] A. Mukhopadhyay and B. W. Sheldon, "Deformation and stress in electrode materials for Li-ion batteries," Prog. Mater. Sci., Vol. 63, no. January, pp. $58-116,2014$

[14] W. Liu, H. Liu, Q. Wang, J. Zhang, B. Xia, and G. Min, "Gas swelling behaviour at different stages in Li4Ti5O12/LiNi1/3Co1/3Mn1/3O2 pouch cells," J. Power Sources, Vol. 369, pp. 103-110, 2017

[15] Y. Li, K. Zhang, B. Zheng, and F. Yang, "Effect of local deformation on the coupling between diffusion and stress in lithium-ion battery," Int. J. Solids Struct., Vol. 87, pp. 81-89, 2016

[16] K. Kanamura, "Electrolytes for lithium batteries," Fluorinated Mater. Energy Convers., No. 6, pp. 253-266, 2005, doi: 10.1016/B978-0080444727/50039-4

[17] Y. Zhan, J. Deng, and T. Wang, "Lithium battery swollen detection based on computer vision," Proc. IEEE Int. Conf. Softw. Eng. Serv. Sci. ICSESS, Vol. 2, pp. 728-731, 2013

[18] B. Bitzer and A. Gruhle, "A new method for detecting lithium plating by measuring the cell thickness," J. Power Sources, Vol. 262, pp. 297-302, 2014

[19] M. Bauer, M. Wachtler, H. Stöwe, J. V. Persson, and M. A. Danzer, "Understanding the dilation and dilation relaxation behavior of graphitebased lithium-ion cells," J. Power Sources, Vol. 317, pp. 93-102, 2016

[20] F. Grimsmann, T. Gerbert, F. Brauchle, A. Gruhle, J. Parisi, and M. Knipper, "Determining the maximum charging currents of lithium-ion cells for small charge quantities," J. Power Sources, Vol. 365, pp. 12-16, 2017

[21] E. Kwak, D. S. Son, S. Jeong, and K. Y. Oh, "Characterization of the mechanical responses of a LiFePO4 battery under different operating conditions," J. Energy Storage, Vol. 28, no. February, 2020 
[22] A. J. Louli, J. Li, S. Trussler, C. R. Fell, and J. R. Dahn, "Volume, Pressure and Thickness Evolution of Li-Ion Pouch Cells with Silicon-Composite Negative Electrodes,” J. Electrochem. Soc., Vol. 164, No. 12, pp. A2689A2696, 2017

[23] B. Rieger, S. V. Erhard, K. Rumpf, and A. Jossen, "A New Method to Model the Thickness Change of a Commercial Pouch Cell during Discharge,” J. Electrochem. Soc., Vol. 163, No. 8, pp. A1566-A1575, 2016

[24] K. Y. Oh, B. I. Epureanu, J. B. Siegel, and A. G. Stefanopoulou, "Phenomenological force and swelling models for rechargeable lithium-ion battery cells," J. Power Sources, Vol. 310, pp. 118-129, 2016

[25] T. Cai, S. Pannala, A. G. Stefanopoulou, and J. B. Siegel, "Battery Internal Short Detection Methodology Using Cell Swelling Measurements," Proc. Am. Control Conf., Vol. 2020-July, pp. 1143-1148, 2020

[26] D. Lu, S. Lin, W. Cui, S. Hu, Z. Zhang, and W. Peng, "Swelling mechanism of $0 \%$ SOC lithium iron phosphate battery at high temperature storage," J. Energy Storage, Vol. 32, no. July, p. 101791, 2020

[27] J. H. Lee, H. M. Lee, and S. Ahn, "Battery dimensional changes occurring during charge/discharge cycles - Thin rectangular lithium ion and polymer cells,” J. Power Sources, Vol. 119-121, pp. 833-837, 2003

[28] B. Sood, C. Hendricks, M. Osterman, and M. Pecht, "Health monitoring of lithium-ion batteries," Electron. Device Fail. Anal., Vol. 16, No. 2, pp. 4-16, 2014

[29] W. Choi, Y. Seo, K. Yoo, T. J. Ko, and J. Choi, "Carbon Nanotube-Based Strain Sensor for Excessive Swelling Detection of Lithium-Ion Battery," $201920^{\text {th }}$ Int. Conf. Solid-State Sensors, Actuators Microsystems Eurosensors XXXIII, TRANSDUCERS 2019 EUROSENSORS XXXIII, no. June, pp. 2356-2359, 2019

[30] D. Sauerteig, S. Ivanov, H. Reinshagen, and A. Bund, "Reversible and irreversible dilation of lithium-ion battery electrodes investigated by in-situ dilatometry," J. Power Sources, Vol. 342, pp. 939-946, 2017

[31] T. Polóni, M. A. Figueroa-Santos, J. B. Siegel, and A. G. Stefanopoulou, "Integration of Non-monotonic Cell Swelling Characteristic for State-ofCharge Estimation,” Proc. Am. Control Conf., Vol. 2018-June, pp. 23062311,2018

[32] Y. Wu et al., "Analysis of manufacturing-induced defects and structural deformations in lithium-ion batteries using computed tomography," Energies, Vol. 11, No. 4, 2018

[33] V. Yufit, P. Shearing, R. W. Hamilton, P. D. Lee, M. Wu, and N. P. Brandon, "Investigation of lithium-ion polymer battery cell failure using X-ray computed tomography," Electrochem. commun., Vol. 13, No. 6, pp. 608- 
610,2011

[34] H. Popp, M. Koller, M. Jahn, and A. Bergmann, "Mechanical methods for state determination of Lithium-Ion secondary batteries: A review," J. Energy Storage, Vol. 32, no. September, p. 101859, 2020

[35] B. Rieger, S. Schlueter, S. V. Erhard, J. Schmalz, G. Reinhart, and A. Jossen, "Multi-scale investigation of thickness changes in a commercial pouch type lithium-ion battery," J. Energy Storage, Vol. 6, pp. 213-221, 2016

[36] M. Gomercic and D. Winter, "Robot-based 3D imaging in industrial inspection," $2^{\text {nd }}$ IEEE Int. Conf. Ind. Informatics, INDIN'04, pp. 421-424, 2004

[37] J. Peterka, L. Morovič, P. Pokorný, M. Kováč, and F. Hornák, "Optical 3D scanning of Cutting tools," Appl. Mech. Mater., Vol. 421, pp. 663-667, 2013

[38] E. M. C. Jones, M. N. Silberstein, S. R. White, and N. R. Sottos, "In Situ Measurements of Strains in Composite Battery Electrodes during Electrochemical Cycling,” Exp. Mech., Vol. 54, No. 6, pp. 971-985, 2014

[39] C. Dai et al., "In situ strain measurements and stress analysis of SiO@C composite electrodes during electrochemical cycling by using digital image correlation," Solid State Ionics, Vol. 331, No. September 2018, pp. 56-65, 2019

[40] P. K. Leung et al., "Real-time displacement and strain mappings of lithiumion batteries using three-dimensional digital image correlation," J. Power Sources, Vol. 271, pp. 82-86, 2014

[41] J. Luo, C.Y. Dai, Z. Wang, K. Liu, W.G. Mao, D.N. Fang, X. Chen, "In-situ measurements of mechanical and volume change of $\mathrm{LiCoO} 2$ lithium-ion batteries during repeated charge-discharge cycling by using digital image correlation", Measurement (2016)

[42] R. Tao, J. Zhu, Y. Zhang, W. L. Song, H. Chen, and D. Fang, "Quantifying the 2D anisotropic displacement and strain fields in graphite-based electrode via in situ scanning electron microscopy and digital image correlation," Extrem. Mech. Lett., Vol. 35, p. 100635, 2020

[43] X. Cheng and M. Pecht, "In situ stress measurement techniques on li-ion battery electrodes: A review," Energies, Vol. 10, No. 5, pp. 1-19, 2017

[44] Y. C. Zhang, O. Briat, J. Y. Deletage, C. Martin, G. Gager, and J. M. Vinassa, "Characterization of external pressure effects on lithium-ion pouch cell,” Proc. IEEE Int. Conf. Ind. Technol., Vol. 2018-Febru, pp. 2055-2059, 2018

[45] C. Parthasarathy, S. Thanagasundaram, and T. K. Jet, "Study of applied pressure on open circuit characteristics and capacity of lithium polymer 
pouch cells," 2016 Asian Conf. Energy, Power Transp. Electrif. ACEPT 2016, 2017

[46] S. Kocsis Szürke and I. Lakatos, "The lithium polymer battery swelling test with high-precision displacement sensors,"2018 20 ${ }^{\text {th }}$ International Symposium on Electrical Apparatus and Technologies (SIELA), Bourgas, 2018, pp. 1-4

[47] Y. L. Dong and B. Pan, "A Review of Speckle Pattern Fabrication and Assessment for Digital Image Correlation," Exp. Mech., Vol. 57, No. 8, pp. 1161-1181, 2017

[48] B. Llamazares, "A Behavioral Analysis of WOWA and SUOWA Operators,” Int. J. Intell. Syst., Vol. 31, No. 8, pp. 827-851, 2016

[49] B. Llamazares, "An analysis of some functions that generalizes weighted means and OWA operators,” Int. J. Intell. Syst., Vol. 28, No. 4, pp. 380-393, 2013

[50] L. A. Zadeh, Studies in Fuzziness and Soft Computing: Foreword, Vol. 261. 2010

[51] E. Damiani, S. De Capitani di Vimercati, P. Samarati, and M. Viviani, "A WOWA-based Aggregation Technique on Trust Values Connected to Metadata," Electron. Notes Theor. Comput. Sci., Vol. 157, No. 3, pp. 131142, 2006

[52] L. Torres, J. C. Becceneri, C. C. Freitas, S. J. S. S. Anna, and S. Sandri, "WOWA image filters."

[53] B. Bede and I. Rudas, "Shooting method for fuzzy two-point boundary value problems," Fuzzy Information Processing Society (NAFIPS), 2012 Annual Meeting of the North American, 2012.08.06-2012.08.08. Berkeley: North American Fuzzy Information Processing Society, pp. 5-8, 2012

[54] C. Carlsson and R. Fuller, Fuzzy Reasoning in Decision Making and Optitnization. Springer-Verlag Berlin Heidelberg, ISBN 978-3-7908-24971,2002

[55] L. Zadeh, "Fuzzy sets," Information and Control, Vol. 8, pp. 338-353, 1965

[56] R. Yager, "On ordered weighted averaging aggregation operators in multicriteria decision making," IEEE Transactions on System, Man and Cybernetics, Vol. 18, pp. 183-190, 1988

[57] G. Beliakov, A. Pradera, and T. Calvo, Aggregation Functions: A Guide for Practitioners. Springer-Verlag Berlin Heidelberg, ISBN 978-3-540-737209, 2007

[58] G. Choquet, “Theory of capacities," Annales de l'Institut Fourier, Vol. 5, pp. 131-154, 1953

[59] V. Torra, "The weighted owa operator," International Journal of Intelligent Systems, Vol. 12, pp. 153-166, 1997 